\title{
Biofilms in 3D porous media: delineating the influence of the pore network geometry, flow and mass transfer on biofilm development
}

\author{
Maxence Carrel ${ }^{\mathrm{a}}$, Verónica L. Morales ${ }^{\mathrm{a}, \mathrm{b}}$, Mario A. Beltran ${ }^{\mathrm{c}, \mathrm{d}}$, Nicolas Derlon ${ }^{\mathrm{a}, \mathrm{e}}$, \\ Rolf Kaufmann ${ }^{\mathrm{d}}$, Eberhard Morgenroth ${ }^{\mathrm{a}, \mathrm{e}}$ and Markus Holzner ${ }^{\mathrm{a}^{*}}$
}

a Institute of Environmental Engineering, ETH Zürich, Stefano-Franscini-Platz 5, 8093 Zürich, Switzerland

$\mathrm{b}$ Department of Civil and Environmental Engineering, University of California, Davis, California, USA

c School of Science, RMIT, Melbourne, Australia

d Empa, Swiss Federal Laboratories for Materials Science and Technology, Center for X-ray Analytics, Dübendorf, Switzerland

e Eawag, Swiss Federal Institute of Aquatic Science and Technology, Dübendorf, Switzerland

Email contact of authors:

holzner@ifu.baug.ethz.ch

${ }^{*}$ Corresponding author

This document is the accepted manuscript version of the following article: Carre1, M., Morales, V. L., Beltran, M. A., Derlon, N., Kaufmann, R., Morgenroth, E., \& Holzner, M. (2018). Biofilms in 3D porous media: delineating the influence of the pore network geometry, flow and mass transfer on biofilm development. Water Research, 134, 280-291. https://doi.org/10.1016/j.watres.2018.01.059 


\section{Biofilm development in a 3D} porous medium

Biofilm adhesion, development and morphology are defined by the local wall shear stress distribution
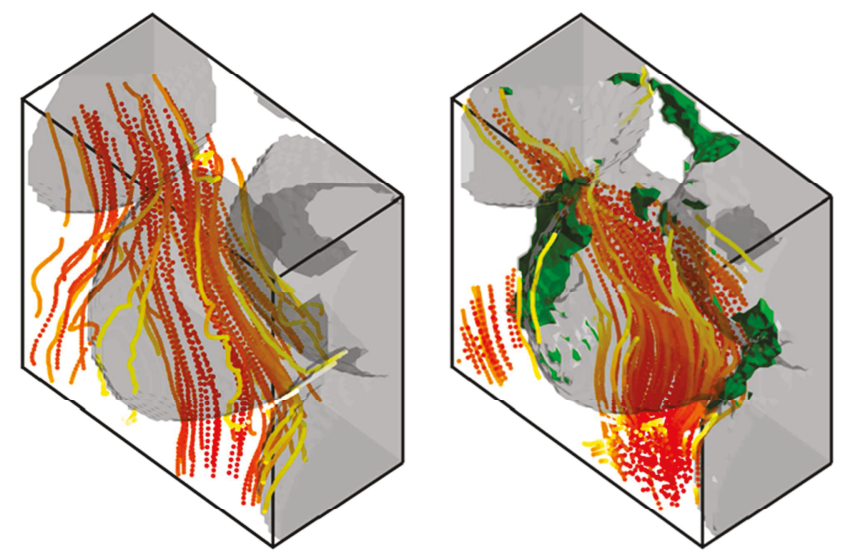


\section{Abstract}

This study investigates the functional correspondence between porescale hydrodynamics, mass transfer, pore structure and biofilm morphology during progressive biofilm colonization of a porous medium. Hydrodynamics and the structure of both the porous medium and the biofilm are experimentally measured with 3D particle tracking velocimetry and micro X-ray Computed Tomography, respectively. The analysis focuses on data obtained in a clean porous medium after $36 \mathrm{~h}$ of biofilm growth. Registration of the particle tracking and X-ray data sets allows to delineate the interplay between porous medium geometry, hydrodynamic and mass transfer processes on the morphology of the developing biofilm. A local analysis revealed wide distributions of wall shear stresses and concentration boundary layer thicknesses. The spatial distribution of the biofilm patches uncovered that the wall shear stresses controlled the biofilm development. Neither external nor internal mass transfer limitations were noticeable in the considered system, consistent with the excess supply of nutrient and electron acceptors. The wall shear stress remained constant in the vicinity of the biofilm but increased substantially elsewhere.

Keywords: biofilm; three-dimensional porous medium; three-dimensional Particle Tracking Velocimetry; X-ray micro Computed Tomography; wall shear stress; concentration boundary layer thickness; biofilm morphology 
58

59

60

61

62

63

64

65

\section{Introduction}

Biofilms are communities of bacteria attaching and developing on surfaces embedded in a matrix of extracellular polymeric substances (EPS) and persistently developing in environmental, medical and industrial settings. In porous media such as soils, many prokaryotic microorganisms develop a sessile lifestyle that allows them to better cope with their environment (Griebler and Lueders 2009). As biofilms are ubiquitous in porous media systems, their relevance stretches over a wide range of applications spanning from the bioremediation of contaminated aquifers (Meckenstock et al. 2015), fixed bed trickling filters (Gujer and Boller 1986, Morgenroth et al. 1996, Gülay et al. 2014) or membrane filtration systems (Baker and Dudley 1998). For all these different applications, biofilm formation can have a positive or detrimental impact. Therefore understanding biofilm development in respect to the local geometry of the pore network, the porescale hydrodynamics and mass transfer processes is a prerequisite for optimal biofilm control. The investigation of these processes requires access to wall shear stresses and concentration boundary layer distributions (Eberl et al. 2000, Picioreanu et al. 2000) based on locally resolved flow and structural information.

The development of biofilms in porous media is a process involving a wide range of scales, from micro- over meso- to macroscale (Battin et al. 2007). For instance, microscale hydrodynamics were shown to control the initial attachment of individual bacteria or particulate matter to surfaces (Rusconi et al. 2014, Radu et al. 2014). The macroscale is the scale relevant for the integrated understanding of larger engineering or natural systems (e.g., reactors, aquifers). The mesoscale links the micro- and the macroscale, as it is the scale at which flow and mass transfer interact with and define the biofilm structures (Eberl et al. 2000). The focus of this study is the mesoscale in porous media or porescale. The processes that drive growth of biofilms are complicated by the complexity of the pore space in which 
they grow in natural porous media, and by the feedback mechanisms between pore clogging from biofilm growth and solute transport (to deliver nutrients and carry away wastes). Subtle changes to the pore structure have been reported to affect pore velocities and characteristic length scales (i.e. pore radii) by orders of magnitude (Seymour et al. 2004, Holzner et al. 2015). Porous media can be considered as networks of connected three-dimensional roughness elements or corners representative of e.g. soils or filters but also of many other pore-scale environments in which biofilms develop.

Biofilm development in porous structures result from highly diverse and complex phenomena. For instance, the growth of biofilms was identified to induce the formation of preferential flow paths, while the interplay between biofilm growth, detachment, decay and lysis was numerically shown to cause the intermittent shifting of these flow paths (Bottero et al. 2013). Locally, the intricate geometry of the pore network and the evolving flow field during biofilm growth influence competition between bacterial communities, as slow growing or non-EPSproducing bacteria can outcompete fast growing or EPS-producing ones (Coyte et al. 2017, Nadell et al. 2017). Various studies of biofilm formation at the porescale mainly considered porous media with two-dimensional pore-networks either experimentally (Drescher et al. 2013, Qian et al. 2017) or numerically (Kapellos et al. 2007). The use of new imaging methods such as Optical Coherence Tomography (OCT) allows linking biofilm formation to local hydrodynamic conditions and overall system performances (Xi et al. 2006, Wagner et al. 2010a, Derlon et al. 2012). OCT allows imaging the biofilm physical structure at the mesoscale. But OCT can also be combined with fluid flow modeling to study flow dynamics (Gao et al. 2014, Weiss et al. 2015). However, the penetration depth of the OCT's signal is limited to around $2 \mathrm{~mm}$ in biofilms, which restrains the application of OCT for monitoring biofilm formation in $3 \mathrm{D}$ porous media. Another relevant method is magnetic resonance imaging (MRI). MRI has been used to investigate transport processes in progressively bioclogged 3D 
porous media at the pore (Seymour et al. 2004) and at the Darcy scale (Codd et al. 2011).

Wagner et al. (2010b) used MRI to study the link between the biofilm formation in a tubular reactor and its influence on the 3D velocity distribution. Results from MRI imaging revealed that biofilm patches could resist maximum local shear stresses up to seven times higher than the mean ones. However, several aspects limit a wide application of MRI to study of biofilm formation in porous media: access to device, cost of the apparatus and use of dedicated flowcells adapted to the MRI, etc. Despite recent progresses, the availability of experimental methods to provide information about both the porescale flow and biofilm development in fully $3 \mathrm{D}$ porous media remains limited.

Experimental data on porescale biofilm properties and hydrodynamics are also required to validate numerical models developed to predict biofilm formation in porous media. A parameter of interest is the biofilm shear strength - the resistance of biofilms to shear exerted by the surrounding fluid. The biofilm shear strength is experimentally challenging to measure and vary with the growth conditions and bacterial type. Yet, many models often use a default value of the biofilms shear strength that may not be representative of their biofilms, thus highlighting the need for direct measurements of biofilm shear strength in 3D porous media. This is reinforced by the wide distributions of biofilm shear strengths mentioned in recent studies (e.g. Stewart (2014)), which can be attributed to the natural heterogeneous distribution of material properties of biofilms (Stewart and Franklin 2008), but also to the distribution of wall shear stresses exerted by fluid flow on biofilms as a consequence of velocity gradients in the biofilm vicinity (Stewart 2014). Additionally, the wall shear stresses exerted on the biofilm are very often roughly approximated based on the initial hydrodynamic conditions (Derlon et al. 2008, Blauert et al. 2015), thus not accounting for the effect of the biofilm formation on the flow, which increases the uncertainty of the assumed biofilm shear strength. 
133 The goal of this paper is thus to experimentally investigate the influence of porescale

hydrodynamics and mass transfer processes (specifically, wall shear stress and concentration boundary layer distribution functions) on biofilm development in a transparent 3D porous media. A biofilm was grown in a 3D porous medium for 36 hours under a constant volumetric flow rate with nutrients and electron acceptors supplied in excess. The hydrodynamics were measured at the porescale with three-dimensional particle tracking velocimetry (3D-PTV) in a clean porous medium and after biofilm growth. The structure of the porous medium, along with the morphology and spatial distribution of the biofilm were obtained with X-ray micro

Computed Tomography (X-ray $\mu \mathrm{CT}$ ). The novel combination of hydrodynamic and structural data permit direct measurements of the feedback mechanisms between biofilm patch development and the fluid dynamics at the porescale to answer the following research questions:

- How does the growth of the biofilm depend on the local wall shear stress and local mass transfer processes?

- What is the influence of the growing biofilm on the porescale hydrodynamics (porescale velocities, wall shear stresses and concentration boundary layer thicknesses)?

\section{Material and Methods}

\subsection{Porous medium}

The porous medium used in this work consists of Nafion pellets (Ion Power, Munich, Germany), a material with physico-chemical properties similar to that of sand grains (Downie et al. 2012). The diameter $d_{N}$ of the pellets is roughly monodisperse and distributed around 2.5 $\pm 0.5 \mathrm{~mm}$. Nafion is an iono-polymer whose optical refractive index can easily be matched (RIM) with aqueous solutions yielding models of transparent soil (Downie et al. 2012). Here, a decent RIM was obtained with a glucose concentration of $11 \% \mathrm{w} / \mathrm{v}$ (see Supplementary 
Information 1 for a detailed analysis concerning the refractive index matching optimization).

The Nafion pellets underwent three times the following treatment allowing optimal transparency. Approximately $20 \mathrm{~g}$. of pellets were heated up at $65^{\circ} \mathrm{C}$ for $1 \mathrm{~h}$ while stirred at $200 \mathrm{rpm}$ under reflux. Afterwards the pellets were cooled for $30 \mathrm{~min}$ at room temperature and stored overnight at $4^{\circ} \mathrm{C}$.

\subsection{Biofilm cultivation}

The $11 \% \mathrm{w} / \mathrm{v}$ glucose solution used as a growth medium in this study was prepared with tap water. In order to enhance the growth of the heterotrophic biofilm cultivated in this experiment, nitrogen and phosphorus were added to a molar ratio C:N:P of 1000:1:1. This low ratio is due to the high glucose concentration that was not only serving as a carbon source but also provided the refractive index matching with the Nafion grains. Nitrate $\left(\mathrm{NaNO}_{3}\right)$ was here serving both as nitrogen source and electron acceptor. Phosphorus was added as $\mathrm{K}_{2} \mathrm{HPO}_{4}$ and $\mathrm{NaH}_{2} \mathrm{PO}_{4} \cdot 2 \mathrm{H}_{2} \mathrm{Oaccounting}$ for $1 / 3$ and $2 / 3$, respectively, of the total phosphorus molecular ratio, yielding inflow concentrations of $8.14 \mathrm{mg} \mathrm{NO}_{3}{ }^{-} \mathrm{-N} / \mathrm{L}$ and $18.9 \mathrm{mg} \mathrm{PO}_{4}{ }^{3-}-\mathrm{P} / \mathrm{L}$.

The mixed species bacterial inoculum used in this study was isolated from the Chriesbach River (Dübendorf, Switzerland, Desmond et al. (2018)). The frozen bacterial inoculum contained in $(2 \mathrm{~mL})$ Eppendorf tubes was added to $100 \mathrm{~mL}$ of the growth medium. It was then incubated for $20-24 \mathrm{~h}$ at $30^{\circ} \mathrm{C}$ and stirred at $200 \mathrm{rpm}$ until reaching midlogarithmic phase $\left(\mathrm{OD}_{600} 0.52 \pm 0.096\right)$. The incubation procedure was repeated three consecutive times for the bacteria to adapt to the synthetic carbon source of the growth medium. For the last incubation cycle, the Nafion grains were added to the growth medium to allow initial bacterial attachment. Upon the incubation, a custom built PMMA flow-cell $\left(38 \times 38 \times 16 \mathrm{~mm}^{3}\right)$ was wet packed with the inoculated Nafion grains. Bottles containing $10 \mathrm{~L}$ of growth medium were connected to the flow cell with silicon tubing (VWR, Dietikon, Switzerland) previously washed with $70 \% \mathrm{v} / \mathrm{v}$ ethanol and thoroughly rinsed with deionized water. The growth 
medium was replaced every $12 \mathrm{~h}$ and spiked with $100 \mathrm{~mL}$ of the inoculum. A peristaltic pump (Ismatec, Glattbrugg, Switzerland) was used to set a volumetric flow rate of $10 \mathrm{~mL} / \mathrm{min}$. As illustrated in Figure 1 (a), a syringe was used as a bubble trap as well as to dampen the pulsatile flow created by the peristaltic pump. Nitrate and oxygen concentration were sampled in the effluent every 12 hours and revealed a high bacterial activity but no actual nutrient limitations (4.41 $\pm 0.67 \mathrm{mg} \mathrm{NO}_{3}{ }^{-} \mathrm{-N} / \mathrm{L}$ and $4.84 \pm 0.55 \mathrm{mg} \mathrm{O}_{2} / \mathrm{L}$ respectively).

\section{Figure 1}

\subsection{Three-Dimensional Particle Tracking Velocimetry}

The three-dimensional particle tracking velocimetry (3D-PTV) method applied in this work allows for detection and tracking of flow particle tracers, which move faithfully with the porewater. Tracking the position of tracer particles provides data on velocity and acceleration along flow trajectories. This method was developed to study turbulent flows (Hoyer et al. 2005) and was lately adapted to study flows in porous media (Holzner et al. 2015). In order to perform 3D-PTV measurements, the flow cell was connected to a $120 \mathrm{~mL}$ syringe mounted on a syringe pump (Lambda Vit-Fit, Lambda, Baar, Switzerland). The volumetric flow rate was set to $10 \mathrm{~mL} / \mathrm{min}$, yielding a Darcy velocity $q$ of $0.27 \mathrm{~mm} / \mathrm{s}$. The estimated Reynolds number was $\operatorname{Re}=q d_{N} / v \approx 0.5$, where $v$ is the kinematic viscosity of the glucose aqueous solution $(v=$ $1.33 \mathrm{e}^{-6} \mathrm{~m}^{2} / \mathrm{s}$ at $\left.20{ }^{\circ} \mathrm{C}\right)$. The tracer particles were composed of fluorescent Red Polyethylene Microspheres (Cospheric, Santa Barbara, CA USA) with a density of $1 \mathrm{~g} / \mathrm{cm}^{3}$ and with a diameter $d_{P}$ of ca. $70 \mu \mathrm{m}(>90 \%$ between 63 and $75 \mu \mathrm{m})$ were added to the growth medium to create a suspension. As these particles are neutrally buoyant, inertial effects are not of concern and the particles follow the flow reliably (Holzner et al. 2015).

For each 3D-PTV measurement, the tracer particle concentration added is of $0.02 \mathrm{~g} / \mathrm{L}$, corresponding to a volume fraction of $0.002 \%$, which is low enough to ensure that particleparticle interactions were not of concern. The fluorescent tracer particles were illuminated 
with a $100 \mathrm{~W}$ pulsed Nd:YLF laser ( $\lambda=527 \mathrm{~nm}$, Darwin Duo, Quantronix, Hamden, USA).

Figure 1 (b) shows the setup used for the 3D-PTV experiments. The flow cell was imaged from both the front and back sides with a Photron Fastcam SA5 with a resolution of $1024 \times 1024$ at 50 frames per second (fps) using an image splitter providing 4 stereoscopic views. Between 30 and 200 particles were tracked per frame, yielding ca. $10^{6}$ data points for every measurement (4549 and 4193 trajectories for the time points $\mathrm{T}=0$ and $\mathrm{T}=36 \mathrm{~h}$ respectively) and 3D particle locations with an accuracy of ca. $50 \mu \mathrm{m}$ (Holzner et al. 2015). Assuming stationarity of the porescale flow and neglecting structural changes induced by biofilm growth during 3D-PTV experiments (ca. $30 \mathrm{~min}$ ), an estimated average inter-particle distance of ca. $50 \mu \mathrm{m}$ was obtained. Additional information about processing of the $3 \mathrm{D}-\mathrm{PTV}$ data and extraction of the wall shear stresses is available in the Supplementary Information 2.

\subsection{X-ray micro-tomography}

Biofilms form porous structures (up to $90 \%$ porosity) (Wagner et al. 2010a) with high water content and densities very close to that of water. Thus, the application of X-ray microtomography to biofilm imaging requires the addition of contrast agents. Here, we follow the approach presented by Davit et al. (2011) and use a suspension of $0.05 \mathrm{~g} / \mathrm{mL}$ barium sulfate $\left(\mathrm{BaSO}_{4}\right)$ particles (Micropaque, Guerbet, Zürich, Switzerland) as a contrast agent that emphasizes the porespace free of biofilm. Davit et al. (2011) noted the occurrence of biofilm detachment occurring during the $\mathrm{BaSO}_{4}$ injection. Carrel et al. (2017) suggested to use iron sulfate $\left(\mathrm{FeSO}_{4}\right)$ as a contrast agent by continuously adding it to the biofilm during culturing and thus avoiding detachment. However, this approach could not be applied here without negatively affecting the RIM. Therefore, $\mathrm{BaSO}_{4}$ was used as a contrast agent. In order to minimize biofilm-contrast agent interactions, the injection of the $\mathrm{BaSO}_{4}$ was done over $12 \mathrm{~h}$ at a volumetric flow rate 500 times smaller than the growth flow rate. 
$\mathrm{X}$-ray $\mu \mathrm{CT}$ scans of the biofilm samples were performed at the Swiss Federal Laboratories for Materials Science and Technology (EMPA, Dübendorf, Switzerland) on a custom-built scanner equipped with a tungsten microfocus source with cone-beam configuration and a $40 \mathrm{x}$ $40 \mathrm{~cm}^{2}$ flat panel detector. Four frames of 1441 projections were acquired over 3 hours at a voltage of $80 \mathrm{kV}$ and focused electron beam current of $125 \mu \mathrm{A}$. Reconstruction was performed as presented in Carrel et al. (2017). The resolution of the obtained tomograms was of $27 \mu \mathrm{m}$. A first scan was imaged prior to the injection of the contrast agent, in order to obtain the structure of the initial porous media. A second scan was imaged after the injection of the contrast agent in order to obtain the biofilm coated porous media.

The reconstructed tomograms exhibited beam-hardening artifacts which were attributed to the polychromatic nature of X-rays, the high absorption coefficient of Barium and the nonhomogeneous distribution of the contrast agent within it. These artifacts were mostly localized near the outermost sides of the anisotropic flow cell. Therefore, a central region of sufficient visualization quality was cropped and used for structural analysis, where the artifacts were weaker (with dimensions of $20 \times 20 \times 16 \mathrm{~mm}$, i.e. $25 \%$ of the total flow cell volume).

Contrast enhancement of the different materials in the tomographic image was effectuated using FIJI (Schindelin et al. 2012). A non-local mean filter was then run in Avizo (Thermo Fisher Scientific, Hillsboro, Oregon, USA) to improve the signal to noise ratio. Segmentation was done using Avizo and consisted of watershed segmentation refined with morphological operations (closing of the solid grains and the biofilm as well as opening of the air bubbles). The air bubbles that entered the flow cell during the injection of the contrast agent were assigned to the liquid phase. Objects smaller than 10 voxels were discarded before the final data evaluation. The procedure presented in Pérez-Reche et al. (2012) was used to measure pore radii along the skeleton of the void space (Additional information concerning the image segmentation is available in the Supplementary Information 3). Bounding boxes fitted to the 
segmented biofilm patches allowed to extract geometric features of the patches such as their aspect ratio, which was obtained by dividing the largest axis of the bounding box by its smallest axis.

\subsection{Registration}

The X-ray segmented data set and the 3D-PTV trajectories were registered (e.g. transformed into one coordinate system) in order to allow a local investigation of the biofilm - flow coupling. The registration was performed using a custom registration algorithm. In a first step, the Lagrangian 3D-PTV flow information was mapped on a Eulerian grid of $81 \mu \mathrm{m}$ size, corresponding to three times the resolution of the X-ray data. The resolution of the X-ray tomograms ( $27 \mu \mathrm{m}$ pixels) was decreased accordingly for the ease of calculation (binning based on voxel averages). Consecutively, a linear transformation was obtained by a discrete pseudo - digital volume correlation maximizing the following criterion:

$$
r_{i j l}=\frac{\sum_{m} \sum_{n} \sum_{o}\left[V_{X}(m+i, n+j, o+l)\right]\left[V_{P}(m, n, o)\right]}{\sum_{m} \sum_{n} \sum_{o}\left[V_{P}(m, n, o)\right]}
$$

where $i, j$, and $l$ are the components of the displacement vector $D(i, j, l), V_{X}$ is the segmented liquid phase of the volumetric X-ray data set and $V_{P}$ is the amount of 3D-PTV Lagrangian data mapped on the Eulerian grid. The final $r_{i j l}$ obtained for the different data sets were of $88.67 \%$ for the clean porous media and of $76.78 \%$ for the bioclogged packing. The uncertainty related to the registration can be inferred to partial volume effects due to the decreased resolution of the tomograms and to the accuracy of the 3D-PTV. Figure 2 (a-d) allows assessing the quality of the registration.

\section{Figure 2}


2.5 Calculation of local wall shear stress and concentration boundary layer thickness

The registered data provided the basis for a local analysis of hydrodynamic and mass transfer processes. A first variable of interest is the wall shear stress $\tau_{w}$, defined as $\tau_{w}=\mu \frac{\partial v}{\partial n}$ where $\mu$ is the dynamic viscosity of the fluid and $\frac{\partial v}{\partial n}$ the velocity gradient defined by the velocity magnitude $v$ and the vector $n$ normal to the triangulated faces of the solid phase (Nafion grains) or to the biofilm surface $(|n|=0)$. In order to evaluate this velocity gradient, the Lagrangian data was first binned on an Eulerian grid of $100 \mu \mathrm{m}$ mesh size. As the interparticle distance of the 3D-PTV data was of ca. $50 \mu \mathrm{m}$ on average, the Eulerian velocity field obtained after binning was not perfectly filled, i.e. there were empty voxels which were not sampled by any fluid particle. The velocity gradients were then interpolated linearly from the Eulerian velocity field on the normal of the solid surface (Nafion grains or biofilm) - that is for all surface patches where velocity information was available - thus providing access to the wall shear stress distribution. Velocity profiles within pores typically exhibit parabolic profiles (de Anna et al. 2017). The accuracy for the wall shear stress was estimated at $8 \%$ by comparing both a linear and a quadratic interpolation of the velocity profile to obtain two different approaches for determining wall shear stress. The comparison between the two interpolation methods indicates that the spatial resolution of the velocity map was sufficient to retrieve wall shear stress with satisfying accuracy. Note that $\tau_{\mathrm{w}}$ was approximated assuming a no-slip boundary condition at the biofilm surface and thus, non-permeable biofilms. However, several authors showed that biofilms are permeable and have networks of submicroscopic channels (Davit et al. 2013, Stoodley et al. 1994). Since the permeability of biofilms is generally fairly low (Deng et al. 2013), we infer that its influence on the approximation of the wall shear stresses is negligible. A second variable of interest that allows assessment of the interplay of mass transfer conditions on biofilm development is the concentration boundary 
302

layer thickness $\delta_{c}$. The mass transfer coefficient $k_{s}=D_{s} / \delta_{c}$ indicates the rate at which substrate or electron acceptors diffuse over the concentration boundary layer thickness $\delta_{c}$ from the bulk of the pore network towards the surface of the grain. Therefore, nutrient limitations are less prone to occur for small concentration boundary layer thicknesses. In order to estimate $\delta_{c}$, we firstly consider that it is linked to the hydraulic boundary layer thickness $\delta_{v}$ as $\delta_{c}=\delta_{v} / S c^{1 / 3}$, where the Schmidt number $S c=v / D$ expresses the ratio of momentum diffusivity $(v)$ to mass diffusivity $(D)$. The thicknesses $\delta_{c}$ and $\delta_{v}$ are commonly defined as lengths stretching normally from the substratum to the 99th percentile of fully developed concentration or velocity profiles respectively. Here, due to the intricate substratum geometries and velocity profiles, the hydraulic boundary layer thickness $\delta_{v}$ is approximated by considering the length scale associated with molecular diffusion of momentum as induced by

shear as $\delta_{v}=\sqrt{v / \frac{\partial v}{\partial n}}$. This means that the concentration boundary layer thickness $\delta_{C}$ is proportional to $\tau_{w}{ }^{-1 / 2}$. Strictly, at locations with negligible biofilm the boundary layer thickness should be very small because no appreciable substratum gradient is present. This implies that using this approach we likely overestimate $\delta_{C}$ in such locations. In the comparative analysis below, we are interested in the dominant factors that control biofilm growth. Hence, our approach provides an estimate of the local $\delta_{C}$ that will develop after biofilm has grown in a given location.

\section{Results}

\subsection{Registered data}

Figure 2 presents the results of the registered 3D-PTV and X-ray data for the central region of the flow cell for the initial clean porous medium $(\mathrm{T}=0 \mathrm{~h})$ in (a) and after $36 \mathrm{~h}$ of biofilm development in (b). Figure 2 (c) and (d) are local close-ups of (a) and (b). The tracer particle trajectories in Figure 2 are color-coded with the velocity magnitude, illustrating the 
intermittency of velocities along trajectories typical of porous media flows (de Anna et al. 2013). The increasingly darker coding of the velocities along trajectories reflects the average velocity increase. Additionally, biofilm growth induced substantial changes on the flow field (compare Figure 2 (a) and (b)), which is restricted to fewer channels.

In Figure 2 (b) biofilm patches are distributed in between Nafion grains. Note that in Figure 2 (b), the flow information is not distributed homogeneously. This could either be caused by flow tracers not sampling stagnation zones or because the view of the particles was obstructed by biofilm patches. The close-ups in Figure 2 (c) and (d) show local changes of the flow field upon biofilm growth. The biofilm patches illustrated in Figure 2 (d) exhibit a high aspect ratio and an orientation approximately aligned with the initial flow direction. Upon biofilm growth, the channel on the left of the central grain presented in Figure 2 (d) appears to be clogged, indicating that growth of a biofilm patch in a pore results in local obstruction of the flow, which consequently is compensated by significant hydrodynamic changes. Figure 2 (e) and (f) present triangulated meshes of the biofilm patches presented in the close-ups and bounding boxes fitted to each individual patch to extract biofilm size and aspect ratio.

\subsection{Influence of biofilm growth on porescale statistics}

In order to quantify the influence of biofilm growth on the porescale hydrodynamics, we conduct a statistical analysis on relevant variables such as the distributions of average porescale velocities, pore radii, wall shear stresses, and concentration boundary layer thicknesses in the clean and bioclogged porous medium. Figure 3 presents the probability density functions (PDFs), which can be considered normalized continuous histograms for the listed variables in the presence and absence of biofilm. Figure 3 (a) presents the PDF of the velocity magnitude for the clean and bioclogged porous medium. Upon biofilm growth, there is a slight increase of the average velocity and a substantial increase of the variance, as seen in the greater frequency in low and high velocities (heavy PDF tailing). These increased tailings 
are typical of flow fields for pore networks of increasing heterogeneity (Morales et al. 2017, Siena et al. 2014). This indicates that the growing biofilm affects the pore structure and leads to the formation of preferential flow paths (increased velocity tails) and slow velocity zones (increased low velocity tail of the PDF). The impact of the biofilm on the pore structure is further confirmed by Figure 3 (b) showing the pore radii distribution for the clean and bioclogged packings. With biofilm growth, the average pore radius decreases from $0.41 \mathrm{~mm}$ to $0.33 \mathrm{~mm}$. Note that these distributions have an exponential tail typical of pore radii distribution in porous media (Holzner et al. 2015).

\section{Figure 3}

The wall shear stress distributions obtained are presented in Figure 3 (c) and span a range of over two orders of magnitude. With biofilm growth, the pore space is reduced and average velocity increased due to mass conservation, while the wall shear stresses increase substantially. Figure 3 (d) shows the distribution of the concentration boundary layer thicknesses $\delta_{c}$ for the clean and bioclogged porous media. As a consequence of the wall shear stress increase observed previously, the concentration boundary layer thickness decreases accordingly.

\subsection{Local statistical analysis}

In this section, we consider the distributions of variables describing the geometry of the pore network and the local hydrodynamics presented in Figure 3. Of interest is the investigation of how these variables locally influence the biofilm or are themselves changed by the developing biofilm. We consider all the points of pore network's skeleton (see Figure 2c) and investigate whether biofilm patches develop in their vicinity (within a distance of one pore radius). This allows us to understand how locally flow and mass transfer influence biofilm development. For the initial time point (at $0 \mathrm{hrs}$ of biofilm growth), this distinction is performed with hindsight for Nafion faces on which biofilm will develop $(B F, 0)$ or those on which it will not 
$376(\mathrm{~N}, 0)$. For the bioclogged data (at $36 \mathrm{hrs}$ of biofilm growth), the distinction is made by classifying surfaces with observable biofilm development $(\mathrm{BF}, 36)$ or Nafion grain surfaces that remained uncolonized $(\mathrm{N}, 36)$.

\section{Figure 4}

Figure 4 (a) and (b) show the PDFs of the porescale velocity magnitude in the vicinity of the developing biofilm (BF) and on uncolonized channels $(\mathrm{N})$ before and after biofilm growth. The PDFs do not show appreciable differences, indicating that the porescale velocities do not directly influence the biofilm development. Figure 4 (c) and (d) show a similar comparison for the pore radii before and after biofilm growth. Here, a noticeable difference emerges, as the pore radii where the biofilm initially develops are on average smaller than in channels where no biofilm grows. Additionally, the pore radii in the biofilm vicinity after $36 \mathrm{~h}$ of growth show a substantial shift in distribution (see Figure 4, d) toward smaller overall pores, particularly in the vicinity of the biofilm. This suggests an increase of the biofilm specific surface area, which is a key parameter for the estimation of mass transfer characteristics within biofilms (Horn and Lackner 2014).

\section{Figure 5}

The distributions of the wall shear stress values obtained for the surface of the clean Nafion grains (solid lines) and for the surface of the nascent biofilm (dashed lines) are shown in Figure 5 at a time prior to biofilm growth (a) and after biofilm growth (b). Although the distribution of wall shear stresses is wide with and without biofilm presence, a strong difference between the two types of surfaces is noticeable. From these data it is possible to note that the maximal wall shear stress for the surfaces that will not be colonized by the biofilm are about twice as large as those where nascent biofilm is found at $36 \mathrm{hr}$. Substantial differences are also observed for the concentration boundary layer thicknesses depicted in Figure 5 (c) and (d). No biofilms were observed to colonize or develop in the high wall shear 
stress regions, despite the small concentration boundary layers present there, corroborating that wall shear stress controls biofilm development in the present experiment. We conjecture from these data that too small pores do have sufficient flux of nutrients to sustain biofilm growth. Mainly, diffusion is the mechanism for nutrient mass transfer, which can be limiting. Too large pores experience high shear, which we interpret to be hydrodynamically unfavourable for biofilms to become established. Presumably the high wall shear detaches nascent colonies and thus prevents significant EPS from developing.

\subsection{Morphology of the biofilm patches}

Figure 6 (a) shows the PDF of the biofilm patch size which follows a power law distribution, where the probability approximately decreases with the inverse of the size. Figure 6 (b) shows the correlation of biofilm patch sizes with average pore radii of the clean porous medium in which the patches grew over the course of the experiment. The biofilm patch size increases with the pore radii, which is expected since the biofilm patches are confined by the radii. The largest biofilm patches are found for average radii of $0.47 \mathrm{~mm}$, slightly larger than the average radius of the porous medium $\left(\left\langle r_{P}\right\rangle=0.41 \mathrm{~mm}\right.$, where the angular brackets denote an average performed over all measured radii). The largest radii appear to be associated with rather small biofilm patches. The wide distribution of the biofilm patch sizes indicates that there is no simple direct relation between the patch size and the pore radii. Figure 6 (c) shows the PDF of the biofilm patches aspect ratio, which is widely distributed and displays a rather high average indicating that elongated shapes are not atypical. Figure 6 (d) shows the correlation of biofilm patch size to aspect ratio, indicating that the maximal size of the biofilm patches decreases with increasing aspect ratio. The PDF of the biofilm thickness, defined as the distance between the biofilm faces to the closest grain faces, is presented in Figure 6 (e). The wide range of biofilm thicknesses observed reflects the patchiness of the biofilm morphologies visible in Figure 3 (e) and (f). Finally, Figure 6 (f) depicts the correlation of biofilm thickness 

with initial wall shear stress, showing that the maximal biofilm thickness tends to decrease with increasing wall shear stresses.

\section{Figure 6}

\subsection{Relation between porescale velocities and radii}

Figure 7 shows joint PDFs of the normalized velocity and of the normalized pore radius for the clean porous medium (a) and the bioclogged medium (b). Holzner et al. (Holzner et al. 2015) conjectured the dependence of the maximum porescale velocity $v_{m}$ on the pore radius $r_{P}$ according to the power law:

$$
v_{m}=v_{0}\left(r_{P} / r_{0}\right)^{\alpha}, \quad-2 \leq \alpha \leq 2
$$

where $v_{0}$ and $r_{0}$ represent characteristic velocities and pore radii. The exponent $\alpha$ is a parameter reflecting the pore network geometry and stretches from -2 for a completely serial pore arrangement (few pathways through which water can flow) to 2 for a completely parallel one (many equally probably pathways for water to flow). The limits to this power law scaling are indicated in Figure 7 as a dashed-dotted and a dashed line. The white circles show the conditional average of $v /<v>$ on $r_{P} /<r_{P}>$ and the continuous line shows a power law fitted to the conditional average. The fitted exponent is noted as imbedded text in the figures. The width of the joint PDFs of $v /\langle v\rangle$ and $r_{P} /\left\langle r_{P}\right\rangle$ increases with biofilm growth, but are found within the scaling corresponding to completely parallel or serial pore arrangements. The exponents measured decrease from 0.257 to 0.063 , reflecting how the pore arrangement changes from parallel towards more serial with biofilm growth.

\section{Figure 7}




\section{Discussion}

\subsection{How does the growth of the biofilm depend on the local wall shear stress and local mass transfer processes?}

The overarching goal of this study is to provide experimental evidence allowing to delineate the influence of porous medium geometry, flow and mass transfer processes on the formation of biofilms in a 3D porous medium. The results obtained indicate that, in 3D porous media representative of some natural and engineering systems, biofilms are exposed to wide distributions of wall shear stresses and concentration boundary layer thicknesses. Biofilm especially tends to develop in low wall shear stress regions (Figure 5 (a) and (b)). After $36 \mathrm{~h}$ of growth, results from X-ray $\mu \mathrm{CT}$ and 3D-PTV revealed that biofilm formation occurred in the regions of low shear stresses, while no or very low biofilm formation was observed in the regions of high shear stresses. Conversely, mass transfer did not seem to play any role, consistent with presence of nutrient and electron acceptors in excess. Had mass transfer been a key variable, we would expect less biofilm to develop in the regions where the concentration boundary layer is thick (see Figure 5 (c) and (d)). It is important to note that this differs, but does not conflict with, studies on bacteria adhesion to clean surfaces, which are found to preferentially accumulate in high shear regions (Rusconi et al., 2014). Those studies focus on the initial adhesion of biofilm forming cells, while the current work concentrates on biofilm development of uniformly attached cells on all grains surfaces, which we observe is shaped by the local hydrodynamics.

The average of the wall shear stress distributions presented in Figure 5 (a) and (b) in the vicinity of the biofilm and at the surface of the bare grains shows a ca. 1.8 fold increase over the course of the experiment (see Table 1). The increase of the maximal wall shear stress measured is substantially higher (2.5) for the bare grains than at the biofilm surface (1.4). Differences in the increase of the maximal values measured for the biofilm compared to the 
471 bare grains suggests that there is a threshold shear stress in the system that the biofilm cannot

472 withstand. This would imply a biofilm shear or cohesive strength of ca. $0.02 \mathrm{~N} / \mathrm{m}^{2}$, which is

473 on the lower end of biofilm shear strengths observed experimentally elsewhere (Stewart 474 2014).

The largest biofilm patch sizes were found in pores of radii close to the average radii, but the wide distribution observed for the patch sizes did not indicate that the local geometry of the pore network was substantially influencing biofilm development. The maximal thickness of the biofilm patches decreased with increasing wall shear stress, showing that for the given porous medium and under the present growth conditions, the wall shear stress played a predominant role on controlling biofilm development.

\subsection{What is the influence of the growing biofilm on the porescale} hydrodynamics

The prevalence of low wall shear stresses in the vicinity of the biofilms could be attributed to the impact of the growing biofilm on the porescale hydrodynamics. Coyte et al. (2017) showed that the local development of a tiny biofilm patch induced an additional pressure drop at a given location of the pore network, which resulted in substantial non-local changes in the overall flow field. In the present study, the flow rate was kept constant over the course of the experiment. Invoking mass conservation and assuming that the biofilm is homogeneously distributed over a typical cylindrical pore (Thullner and Baveye 2008) would mean that the average velocity would increase in an inverse quadratic relation relative to the pore radius $\left(v \propto Q /\left(\pi r^{2}\right)\right.$ (see dashed-dotted lines on Figure 7). However, as the data presented in this study show, the biofilm is not homogeneously distributed at the grain surface. Furthermore, even if the flow rate were kept constant such that the pressure gradient increased at the flow cell scale, locally, it is possible to have zones with only small pressure gradient variations. For 
similar pressure gradients, according to Poiseuille's law, the velocity is proportional to the square of the radius ( $v \propto r^{2}$ see dashed lines on Figure 7), so that low velocity regions or stagnant zones can form with biofilm growth. These considerations suggest that predicting the local impact of the growth of biofilm on the porescale hydrodynamics is far from trivial, as for example, porescale velocities could increase or decrease with the pore radius variation. The complex interactions between biofilm development and porescale hydrodynamics is illustrated by the radius-velocity relation presented in Figure 7. The experimental data presented in this study shows that there is a formation of high velocity regions, as also indicated by the high velocity tails of the velocity magnitude PDFs (Figure 3 a).

\subsection{Significance}

To our knowledge, our work is one of the first experimental studies of the biofilm shear strength in three-dimensional porous media. It is important to note that the small wall shear stress values obtained here are of the same order of magnitude than values obtained elsewhere for low Reynolds number flows (Nadell et al. 2017, Song et al. 2014). If we consider variability in shear strengths from different types of biofilms, the direct measurements of wall shear strength presented here are on the lower end of values reported in other studies $(0.1$ to more than $10^{\prime} 000 \mathrm{~N} / \mathrm{m}^{2}$ as reported in Möhle et al. (2007), Derlon et al. (2008) or Stewart (2014)). The ability of measuring in-situ the biofilm shear strength in 3D porous media opens several research opportunities, namely to use this approach for the validation of numerical models.

Numerical models often rely on default values of biofilm shear strengths obtained from the literature (Bottero et al. 2013, Pintelon et al. 2012). Our results, however, underline the risk associated with using generic values for the biofilm shear strength, because these values might relate to non-relevant growth conditions and thus overestimate this system parameter. 
The experimental method introduced in our study allows to access realistic biofilm shear strengths in $3 \mathrm{D}$ porous media with an accuracy of about $8 \%$. We suggest that this approach can hence be used to validate assumptions made for numerical models of biofilm formation in 3D porous media providing distributions of wall shear stress values local to the biofilm.

Given that both optical camera setups and laboratory X-ray scanners become more and more a standard equipment of many laboratories, our approach offers a viable method to resolve the geometry of the porous media in conjunction with the biofilm morphology as well as distribution of the local wall shear stresses. A limitation of our method is that the X-rays might inactivate cells of the biofilm, which would impact subsequent measurements of biofilm morphology. In this study, we have analyzed only one bioclogged state $(\mathrm{T}=36 \mathrm{~h})$ and we have not quantified this possible influence, which therefore remains a subject of future work. Possible alternative methods, which would not suffer from this limitation, are based on magnetic resonance microscopy adapting the approach introduced by (Wagner et al. 2010b) to 3D porous media or by combining 3D geometries obtained from X-ray micro-tomography with numerical studies of coupled flow and biofilm growth (Peszynska et al. 2016). Each of these approaches (PTV-X-ray combination, magnetic resonance and X-ray - numerical simulation combination) has its own strengths and weaknesses with respect to spatial resolution, accuracy and assumptions made concerning biofilm-flow coupling. The magnetic resonance approach offers even finer $(<50 \mu \mathrm{m})$ spatial resolution than the present approach and does not require index matching or referencing between flow and biofilm data because all phases are extracted from the same data set. However, it is not trivial to distinguish between flow in pore spaces and fluid inside biofilms because biofilms tend to be permeable and the magnetic resonance signal also captures flow inside the biofilm (e.g. Seymour et al. 2004). Numerical simulations based on X-ray geometries offer very high resolution and accuracy. However, assumptions must be made, for example, concerning the permeability of the biofilm 
or biofilm growth (e.g. von Schulenburg et al. 2009, Bottero et al. 2013). There is therefore no best approach but rather the optimal method should be chosen depending on the specific setup and research goal in mind.

\section{Conclusions}

This study presents experimental measurements on porescale hydrodynamics, porespace structure and biofilm morphology in a progressively bioclogged porous medium with the aim of delineating the influence of the porespace geometry, wall shear stresses, and mass transfer processes on biofilm growth. The local wall shear stress measurements revealed that the attachment and development of biofilm patches was controlled by the local wall shear stress. Biofilm formation occurred in the regions of low shear stresses, while no significant amount of biofilm grew in the regions of high shear stresses. Biofilms were found at local wall shear stresses up to $0.02 \mathrm{~N}$, which defines a maximal shear strength of the biofilm in the porous medium. Mass transfer processes played a secondary role for growth, consistent with presence of nutrient and electron acceptors in excess in our experiments. The development of biofilm substantially influenced the porescale hydrodynamics, as shown by the significant increase of the pore velocities and wall shear stresses, both in terms of average value and variance. Given that the flow rate was kept constant, the increase of average pore velocity is a consequence of the porosity reduction upon biofilm growth. However, growth was not homogeneous in space and resulted in the clogging of certain pores so that the pore scale flow arrangement changed from a predominantly parallel configuration towards a more serial one. This is a manifestation of the formation of preferential flow pathways in the bioclogged porous medium. We propose that, based on this method, measured biofilm shear strength could be used to validate models used in numerical simulations of biofilm growth in porous media. Furthermore, the growth conditions used in the current study could be tailored to provide experimental data for practical applications that seek to optimize biofilm thickness or specific surface area and that 
570 upscale mass transfer processes to practically relevant scales of sand or trickling filters.

571 Finally, with the fast pace of development of 3D printing technology (in terms of printable

572 materials, geometries and scales accessible), the 3D printing of Nafion (James et al. 2015)

573 might soon allow similar investigations in other geometries (non-granular porous media,

574 membrane feed spacer channels) and at scales relevant for other practical applications.

\section{Acknowledgements}

576 We thank Toni Blunschi for manufacturing the flow cells, Daniel Braun, Lucien Biolley and

577 Ela Burmeister for providing some of the hardware necessary for this study and Peter

578 Desmond for sharing the bacteria cultures. We acknowledge the contribution of Andris Wyss

579 in the frame of a semester project. The authors thank Matthias Willmann for stimulating

580 discussions and Stefan Hartmann for his help with the X-ray $\mu$ CT measurements. Part of this

581 work has been performed using the Empa Platform for Image Analysis

582 (http://empa.ch/web/s499/software-/-imaging-platform) at Empa's Center for X-ray Analytics.

583 Financial support is gratefully acknowledged from the Swiss National Science Foundation

584 (SNF grant number 144645 and 172916) for M.C. and M.H. as well as a SNF mobility grant

585 for doctoral students for M.C.. V.L.M. acknowledges the financial support of the AXA

586 Research fund. 
589

590

591

592

593

594

595

596

597

598

599

600

601

602

603

604

605

606

607

608

609

610

611

612

613

614

615

616

617

618

619

620

621

622

623

624

625

626

627

628

629

630

631

632

633

634

635

636

637

638

Griebler, C. and Lueders, T. (2009) Microbial biodiversity in groundwater ecosystems. Freshwater Biology 54(4), 649-677.

Meckenstock, R.U., Elsner, M., Griebler, C., Lueders, T., Stumpp, C., Aamand, J., Agathos, S.N., Albrechtsen, H.-J., Bastiaens, L., Bjerg, P.L., Boon, N., Dejonghe, W., Huang, W.E., Schmidt, S.I., Smolders, E., Sørensen, S.R., Springael, D. and van Breukelen, B.M. (2015) Biodegradation: Updating the Concepts of Control for Microbial Cleanup in Contaminated Aquifers. Environmental Science \& Technology 49(12), 7073-7081.

Gujer, W. and Boller, M. (1986) Design of a nitrifying tertiary trickling filter based on theoretical concepts. Water Research 20(11), 1353-1362.

Morgenroth, E., Schroeder, E.D., Chang, D.P.Y. and Scow, K.M. (1996) Nutrient Limitation in a Compost Biofilter Degrading Hexane. Journal of the Air \& Waste Management Association 46(4), 300308.

Gülay, A., Tatari, K., Musovic, S., Mateiu, R.V., Albrechtsen, H.-J. and Smets, B.F. (2014) Internal Porosity of Mineral Coating Supports Microbial Activity in Rapid Sand Filters for Groundwater Treatment. Applied and Environmental Microbiology 80(22), 7010-7020.

Baker, J.S. and Dudley, L.Y. (1998) Biofouling in membrane systems - A review. Desalination 118(1), 81-89.

Eberl, H.J., Picioreanu, C., Heijnen, J.J. and van Loosdrecht, M.C.M. (2000) A three-dimensional numerical study on the correlation of spatial structure, hydrodynamic conditions, and mass transfer and conversion in biofilms. Chemical Engineering Science 55(24), 6209-6222.

Picioreanu, C., van Loosdrecht, M.C.M. and Heijnen, J.J. (2000) A theoretical study on the effect of surface roughness on mass transport and transformation in biofilms. Biotechnology and Bioengineering 68(4), 355-369.

Battin, T.J., Sloan, W.T., Kjelleberg, S., Daims, H., Head, I.M., Curtis, T.P. and Eberl, L. (2007) Microbial landscapes: new paths to biofilm research. Nat Rev Micro 5(1), 76-81.

Rusconi, R., Guasto, J.S. and Stocker, R. (2014) Bacterial transport suppressed by fluid shear. Nat Phys 10(3), 212-217.

Radu, A., van Steen, M., Vrouwenvelder, J.S., van Loosdrecht, M. and Picioreanu, C. (2014) Spacer geometry and particle deposition in spiral wound membrane feed channels. Water Research 64, 160176.

Seymour, J.D., Gage, J.P., Codd, S.L. and Gerlach, R. (2004) Anomalous Fluid Transport in Porous Media Induced by Biofilm Growth. Physical Review Letters 93(19), 198103.

Holzner, M., Morales, V.L., Willmann, M. and Dentz, M. (2015) Intermittent Lagrangian velocities and accelerations in three-dimensional porous medium flow. Physical Review E 92(1), 013015.

Bottero, S., Storck, T., Heimovaara, T.J., van Loosdrecht, M.C.M., Enzien, M.V. and Picioreanu, C. (2013) Biofilm development and the dynamics of preferential flow paths in porous media. Biofouling. Coyte, K.Z., Tabuteau, H., Gaffney, E.A., Foster, K.R. and Durham, W.M. (2017) Microbial competition in porous environments can select against rapid biofilm growth. Proceedings of the National Academy of Sciences 114(2), E161-E170.

Nadell, C.D., Ricaurte, D., Yan, J., Drescher, K. and Bassler, B.L. (2017) Flow environment and matrix structure interact to determine spatial competition in Pseudomonas aeruginosa biofilms. eLife 6 , e21855.

Drescher, K., Shen, Y., Bassler, B.L. and Stone, H.A. (2013) Biofilm streamers cause catastrophic disruption of flow with consequences for environmental and medical systems. Proceedings of the National Academy of Sciences 110(11), 4345-4350.

Qian, J., Horn, H., Tarchitzky, J., Chen, Y., Katz, S. and Wagner, M. (2017) Water quality and daily temperature cycle affect biofilm formation in drip irrigation devices revealed by optical coherence tomography. Biofouling 33(3), 211-221.

Kapellos, G.E., Alexiou, T.S. and Payatakes, A.C. (2007) Hierarchical simulator of biofilm growth and dynamics in granular porous materials. Advances in Water Resources 30(6-7), 1648-1667. 
Xi, C., Marks, D., Schlachter, S., Luo, W. and Boppart, S.A. (2006) High-resolution three-dimensional imaging of biofilm development using optical coherence tomography. Journal of Biomedical Optics 11(3), 034001-034001-034006.

Wagner, M., Taherzadeh, D., Haisch, C. and Horn, H. (2010a) Investigation of the mesoscale structure and volumetric features of biofilms using optical coherence tomography. Biotechnology and Bioengineering 107(5), 844-853.

Derlon, N., Peter-Varbanets, M., Scheidegger, A., Pronk, W. and Morgenroth, E. (2012) Predation influences the structure of biofilm developed on ultrafiltration membranes. Water Research $46(10)$, 3323-3333.

Gao, Y., Haavisto, S., Li, W., Chuyang, T., Salmela, J. and Fane, A.G. (2014) A Novel Approach to Characterizing the Growth of a Fouling Layer during Membrane Filtration via Optical Coherence Tomography. Environmental Science \& Technology.

Weiss, N., van Leeuwen, T.G. and Kalkman, J. (2015) Simultaneous and localized measurement of diffusion and flow using optical coherence tomography. Optics Express 23(3), 3448-3459.

Codd, S.L., Vogt, S.J., Hornemann, J.A., Phillips, A.J., Maneval, J.E., Romanenko, K.R., Hansen, L., Cunningham, A.B. and Seymour, J.D. (2011) NMR relaxation measurements of biofouling in model and geological porous media. Organic Geochemistry 42(8), 965-971.

Wagner, M., Manz, B., Volke, F., Neu, T.R. and Horn, H. (2010b) Online assessment of biofilm development, sloughing and forced detachment in tube reactor by means of magnetic resonance microscopy. Biotechnology and Bioengineering 107(1), 172-181.

Stewart, P.S. (2014) Biophysics of biofilm infection. Pathogens and Disease 70(3), 212-218. Stewart, P.S. and Franklin, M.J. (2008) Physiological heterogeneity in biofilms. Nat Rev Micro 6(3), 199-210.

Derlon, N., Massé, A., Escudié, R., Bernet, N. and Paul, E. (2008) Stratification in the cohesion of biofilms grown under various environmental conditions. Water Research 42(8-9), 2102-2110.

Blauert, F., Horn, H. and Wagner, M. (2015) Time-resolved biofilm deformation measurements using optical coherence tomography. Biotechnology and Bioengineering 112(9), 1893-1905.

Downie, H., Holden, N., Otten, W., Spiers, A.J., Valentine, T.A. and Dupuy, L.X. (2012) Transparent Soil for Imaging the Rhizosphere. PLoS ONE 7(9), e44276.

Desmond, P., Best, J.P., Morgenroth, E. and Derlon, N. (2018) Linking composition of extracellular polymeric substances (EPS) to the physical structure and hydraulic resistance of membrane biofilms. Water Research 132, 211-221.

Hoyer, K., Holzner, M., Lüthi, B., Guala, M., Liberzon, A. and Kinzelbach, W. (2005) 3D scanning particle tracking velocimetry. Experiments in Fluids 39(5), 923-934.

Davit, Y., Iltis, G., Debenest, G., Veran-Tissoires, S., Wildenschild, D., Gerino, M. and Quintard, M. (2011) Imaging biofilm in porous media using X-ray computed microtomography. Journal of Microscopy 242(1), 15-25.

Carrel, M., Beltran, M.A., Morales, V.L., Derlon, N., Morgenroth, E., Kaufmann, R. and Holzner, M. (2017) Biofilm imaging in porous media by laboratory X-Ray tomography: Combining a nondestructive contrast agent with propagation-based phase-contrast imaging tools. PLOS ONE 12(7), e0180374.

Schindelin, J., Arganda-Carreras, I., Frise, E., Kaynig, V., Longair, M., Pietzsch, T., Preibisch, S., Rueden, C., Saalfeld, S., Schmid, B., Tinevez, J.-Y., White, D.J., Hartenstein, V., Eliceiri, K., Tomancak, P. and Cardona, A. (2012) Fiji: an open-source platform for biological-image analysis. Nature Methods 9 , 676.

Pérez-Reche, F.J., Taraskin, S.N., Otten, W., Viana, M.P., Costa, L.d.F. and Gilligan, C.A. (2012) Prominent Effect of Soil Network Heterogeneity on Microbial Invasion. Physical Review Letters 109(9), 098102.

de Anna, P., Quaife, B., Biros, G. and Juanes, R. (2017) Prediction of the low-velocity distribution from the pore structure in simple porous media. Physical Review Fluids 2(12), 124103.

Davit, Y., Byrne, H., Osborne, J., Pitt-Francis, J., Gavaghan, D. and Quintard, M. (2013) Hydrodynamic dispersion within porous biofilms. Physical Review E 87(1), 012718. 
Stoodley, P., deBeer, D. and Lewandowski, Z. (1994) Liquid Flow in Biofilm Systems. Applied and Environmental Microbiology 60(8), 2711-2716.

Deng, W., Cardenas, M.B., Kirk, M.F., Altman, S.J. and Bennett, P.C. (2013) Effect of Permeable Biofilm on Micro- And Macro-Scale Flow and Transport in Bioclogged Pores. Environmental Science \& Technology 47(19), 11092-11098.

de Anna, P., Le Borgne, T., Dentz, M., Tartakovsky, A.M., Bolster, D. and Davy, P. (2013) Flow Intermittency, Dispersion, and Correlated Continuous Time Random Walks in Porous Media. Physical Review Letters 110(18), 184502.

Morales, V.L., Dentz, M., Willmann, M. and Holzner, M. (2017) Stochastic dynamics of intermittent pore-scale particle motion in three-dimensional porous media: Experiments and theory. Geophysical Research Letters, n/a-n/a.

Siena, M., Riva, M., Hyman, J.D., Winter, C.L. and Guadagnini, A. (2014) Relationship between pore size and velocity probability distributions in stochastically generated porous media. Physical Review $\mathrm{E}$ 89(1), 013018.

Horn, H. and Lackner, S. (2014) Productive Biofilms. Muffler, K. and Ulber, R. (eds), pp. 53-76, Springer International Publishing, Cham.

Thullner, M. and Baveye, P. (2008) Computational pore network modeling of the influence of biofilm permeability on bioclogging in porous media. Biotechnology and Bioengineering 99(6), 1337-1351. Song, J.L., Au, K.H., Huynh, K.T. and Packman, A.I. (2014) Biofilm responses to smooth flow fields and chemical gradients in novel microfluidic flow cells. Biotechnology and Bioengineering 111(3), 597607.

Möhle, R.B., Langemann, T., Haesner, M., Augustin, W., Scholl, S., Neu, T.R., Hempel, D.C. and Horn, H. (2007) Structure and shear strength of microbial biofilms as determined with confocal laser scanning microscopy and fluid dynamic gauging using a novel rotating disc biofilm reactor. Biotechnology and Bioengineering 98(4), 747-755.

Pintelon, T.R.R., Picioreanu, C., van Loosdrecht, M.C.M. and Johns, M.L. (2012) The effect of biofilm permeability on bio-clogging of porous media. Biotechnology and Bioengineering 109(4), 1031-1042. Peszynska, M., Trykozko, A., Iltis, G., Schlueter, S. and Wildenschild, D. (2016) Biofilm growth in porous media: experiments, computational modeling at the porescale, and upscaling. Advances in Water Resources 95, 288-301. 
723 List of tables:

724 Table 1

725 


\section{ACCEPTED MANUSCRIPT}

726 Table 1: Average and maximal values of the wall shear stress measured at the location of the nascent biofilm and at the surface of the biofilm as well as at the surface of the solid grains at

728 the start and end of the experiment. The distinction was here performed by considering the

729 data in one radius distance of the solid (Nafion grains $(\mathrm{N})$ or biofilm (BF) faces). $\mathrm{I}_{\mathrm{F}}$ stands for

730 the increase factor between the initial corresponding value and the value obtained after

731 biofilm growth.

\begin{tabular}{lcccccc}
\hline & \multicolumn{3}{c}{$<\tau_{w}>\left(\mathrm{N} / \mathrm{m}^{2}\right)$} & \multicolumn{3}{c}{$\max \left(\tau_{w}\right)\left(\mathrm{N} / \mathrm{m}^{2}\right)$} \\
& $\mathrm{T}=0 \mathrm{~h}$ & $\mathrm{~T}=36 \mathrm{~h}$ & $\mathrm{I}_{\mathrm{F}}$ & $\mathrm{T}=0 \mathrm{~h}$ & $\mathrm{~T}=36 \mathrm{~h}$ & $\mathrm{I}_{\mathrm{F}}$ \\
\hline $\mathrm{BF}$ & 0.0037 & 0.0067 & 1.8 & 0.0152 & 0.0214 & 1.4 \\
$\mathrm{~N}$ & 0.0065 & 0.0129 & 1.9 & 0.0391 & 0.0981 & 2.5 \\
\hline
\end{tabular}

732

733

734

735

736

737

738

739 
$740 \quad$ List of figures:

$741 \quad$ Figure 1

$742 \quad$ Figure 2

$743 \quad$ Figure 3

$744 \quad$ Figure 4

$745 \quad$ Figure 5

$746 \quad$ Figure 6

$747 \quad$ Figure 7

748 
(a)

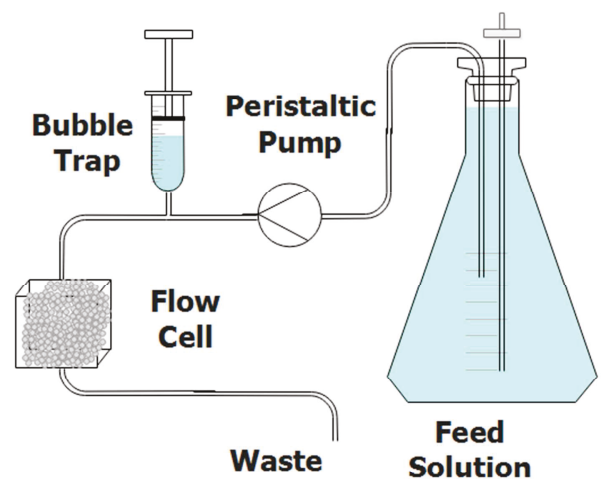

(b)

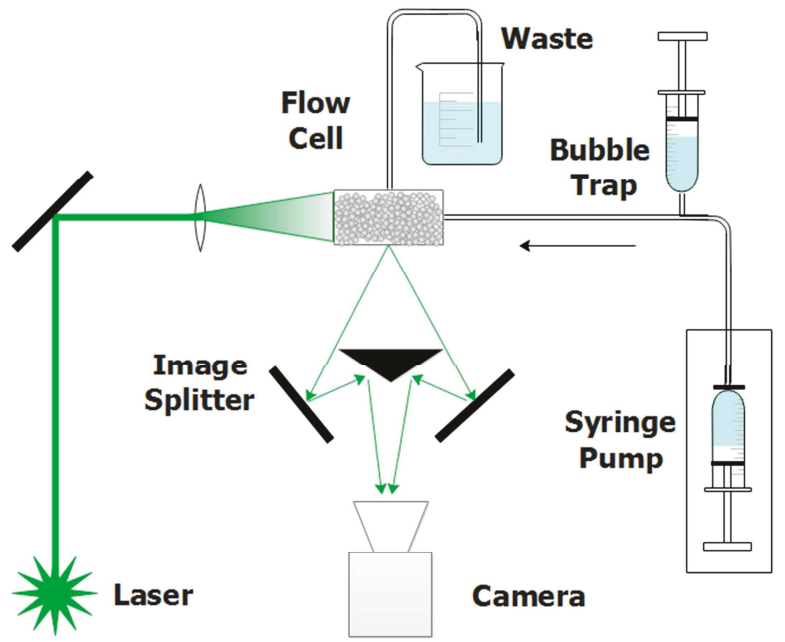

750 Figure 1: Schematics of the set up used (a) for biofilm cultivation and (b) for the 3D-PTV measurements. 


\section{ACCEPTED MANUSCRIPT}

753

754

755

756

757

758

759

760

761

762

763

764

765

766

767

768

769

770

771

772

773

774

775

776

777

778 (a)

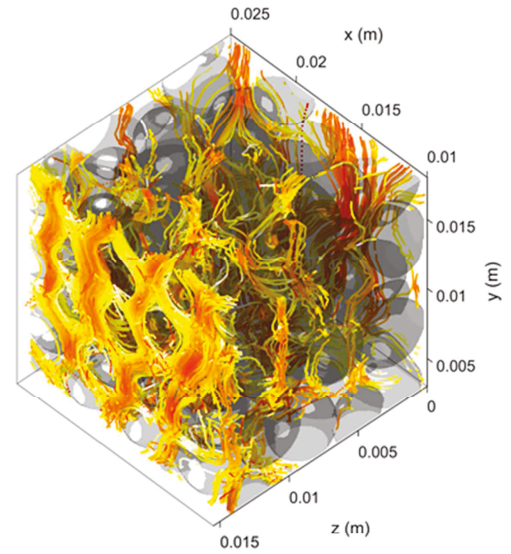

(c)

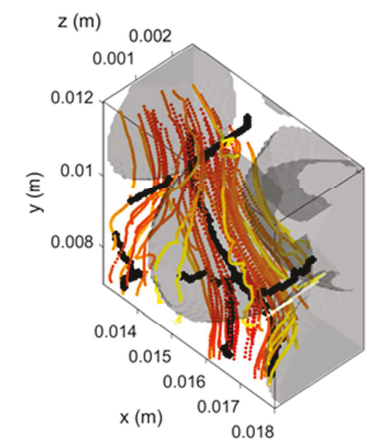

(e)

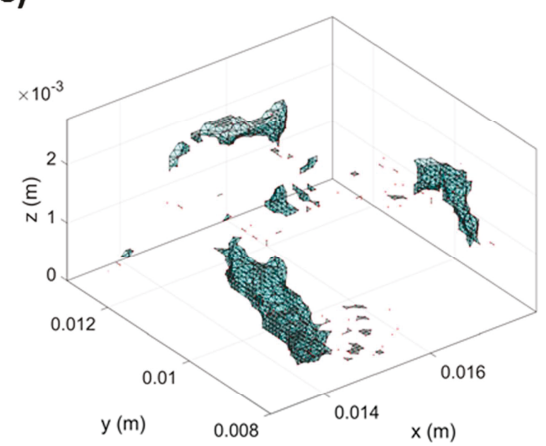

(b)

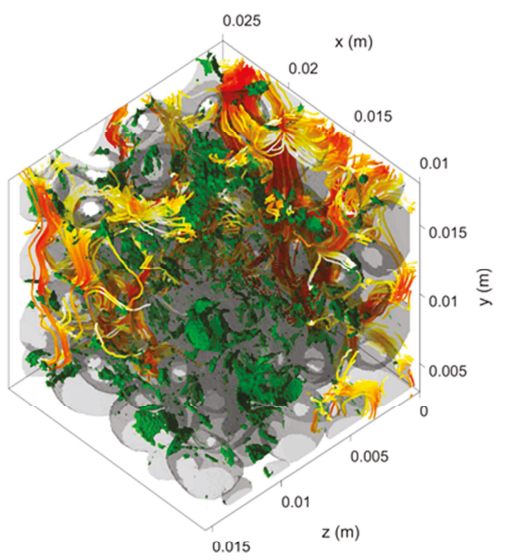

(d)

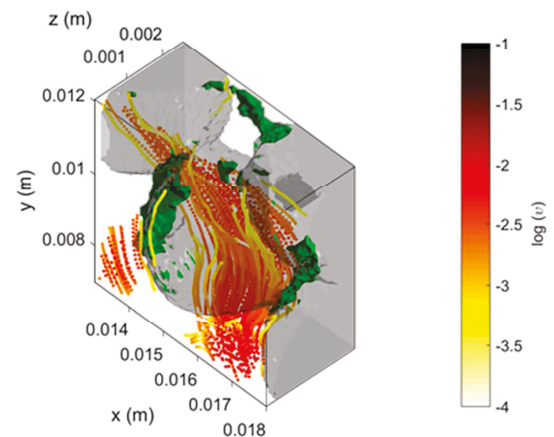

(f)

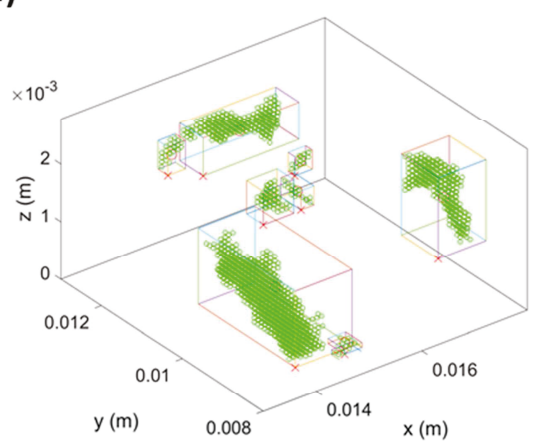

Figure 2: Registered 3D-PTV and X-ray data of the central zone for the clean (a) and bioclogged (b) porous medium. The solid surfaces (Nafion grains and biofilm) represent a color-coded (Nafion grains in grey and biofilm in green) Delaunay triangulation of the segmented X-ray data. (c) and (d) show a local magnification of a pore before and after biofilm colonization. Black lines in (c) represent the skeleton along which the pore radii were computed. The colorbar in (d) shows the scale of the velocity magnitude used for (a)-(d). The pore skeleton is not shown in (a), (b) and (d) for the sake of clarity. (e) shows the biofilm patches illustrated in (d). (f) shows the same patches and bounding boxes from which the aspect ratio of the patches were computed. Objects smaller than 10 voxels visible in (e) are removed in (f). 
(a)

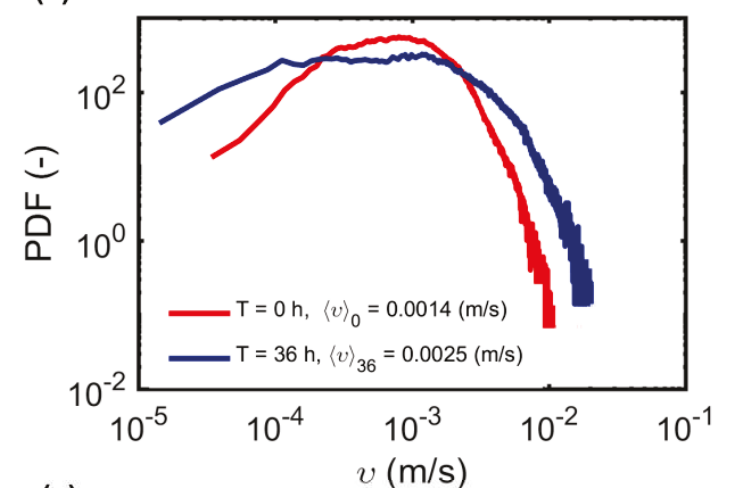

(c)

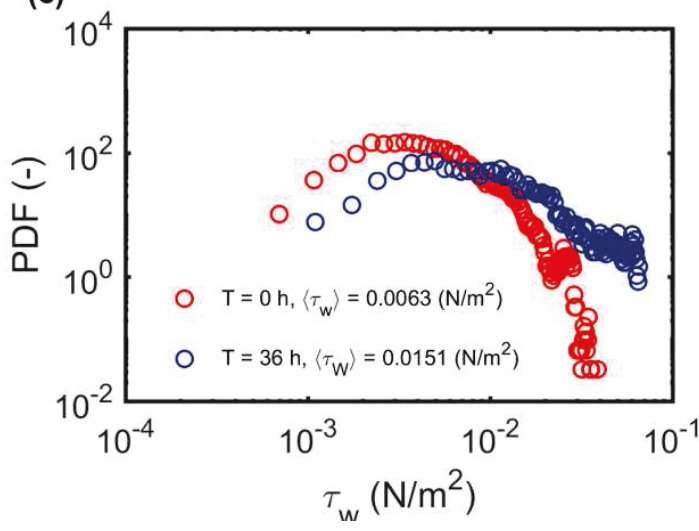

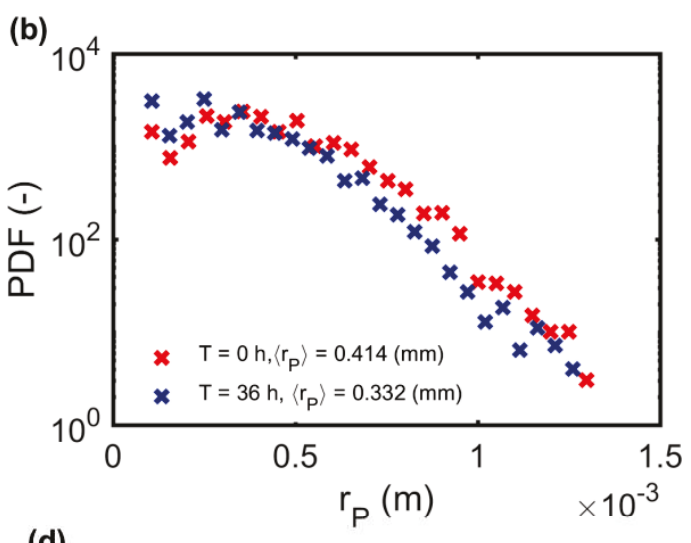

(d)

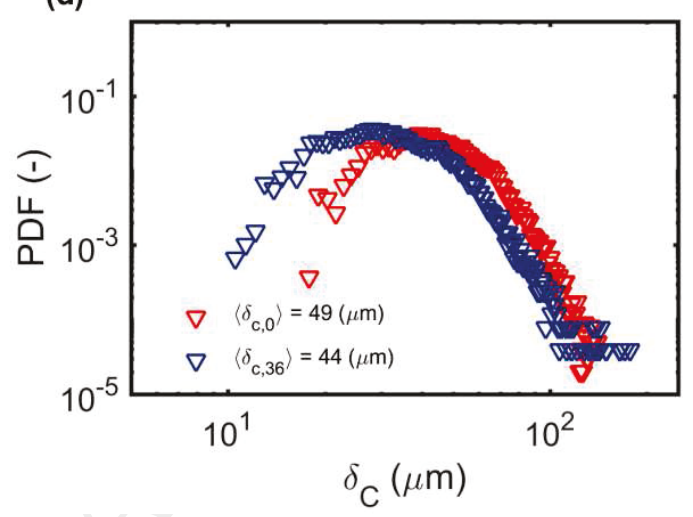

Figure 3: Probability density functions of the velocity magnitude (a), pore radii (b), wall

781

782

783

784

785

786

787

shear stresses (c), and of concentration boundary layer thicknesses (d) for the two different

time points during biofilm growth: clean $(\mathrm{T}=0 \mathrm{hr})$ and bioclogged porous media $(\mathrm{T}=36 \mathrm{~h})$. Angular brackets denote the average over all measured values. 
(a)

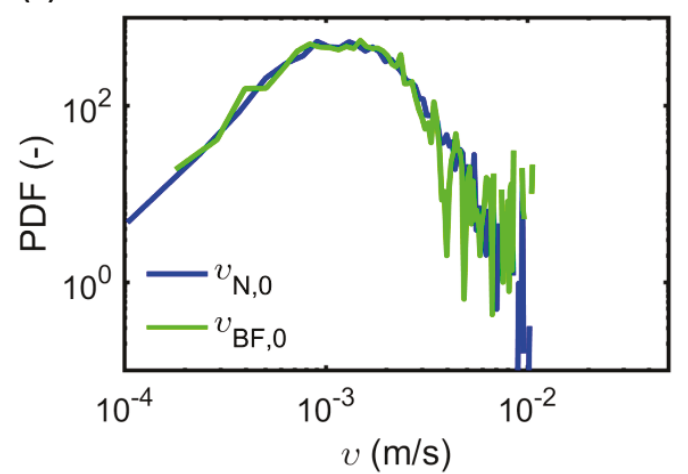

(c)

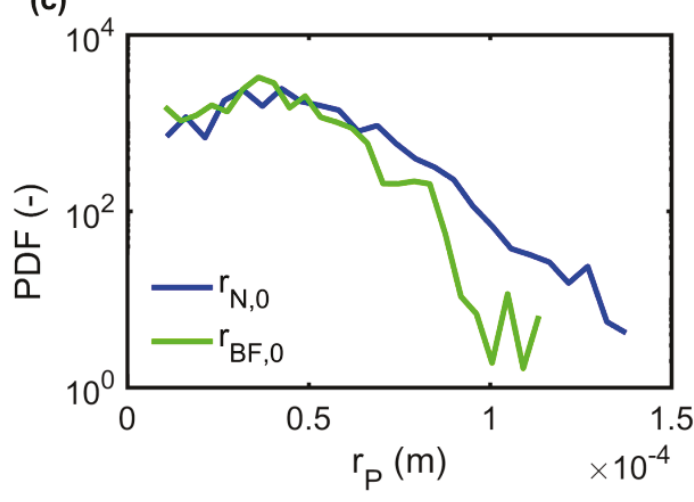

(b)

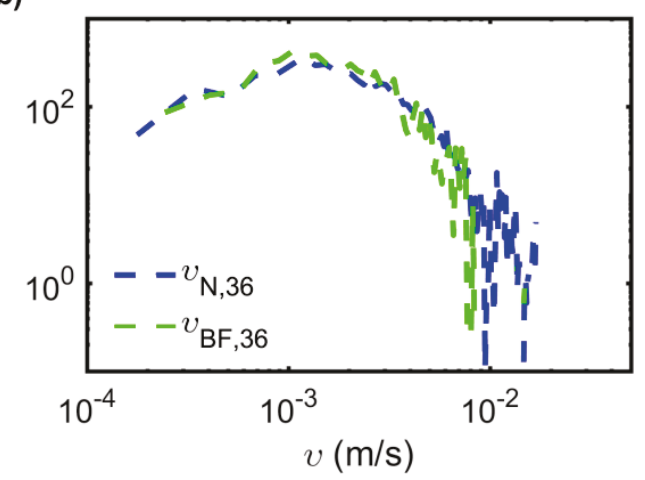

(d)

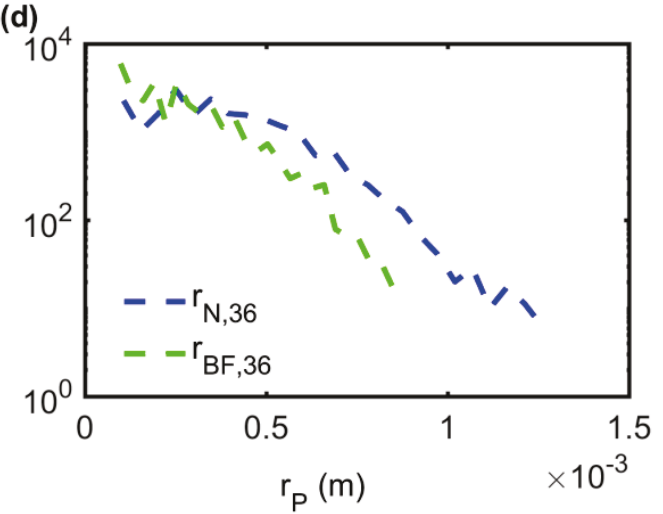

789 Figure 4: (a) and (b) show the PDFs of the pore-scale velocity magnitudes for the clean and 790 bioclogged porous medium, whereas (c) and (d) show the corresponding pore radii PDFs.

791 . "BF" and "N" here indicates if the considered data is located within a distance of one pore 792 radius to the biofilm or uncolonized Nafion grains, respectively. 
(a)

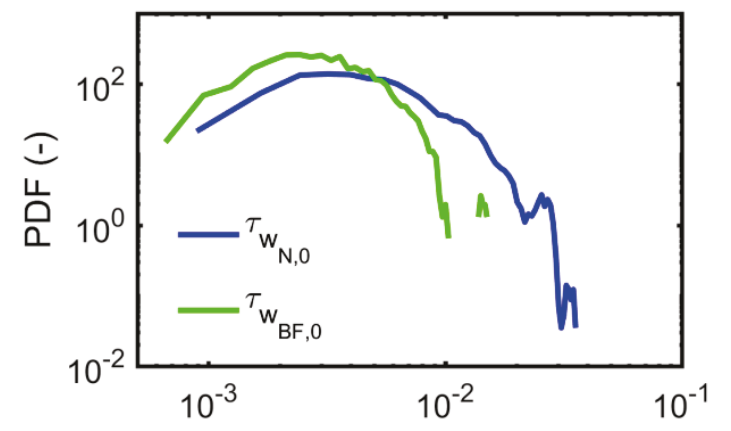

(c) $\quad \tau_{\mathrm{w}}\left(\mathrm{N} / \mathrm{m}^{2}\right)$

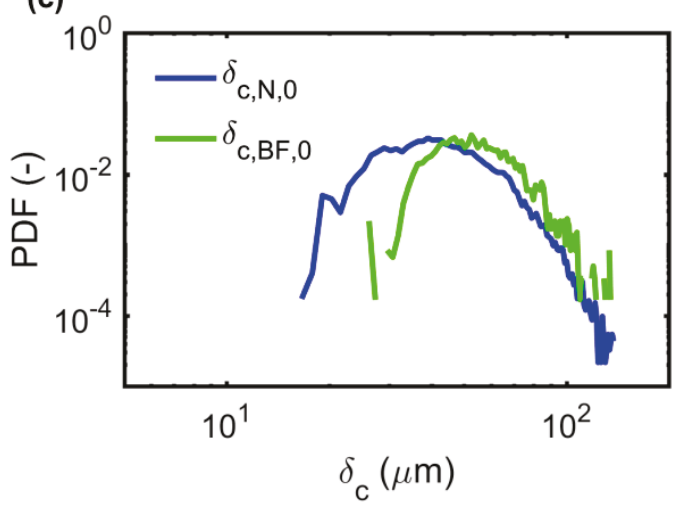

(b)

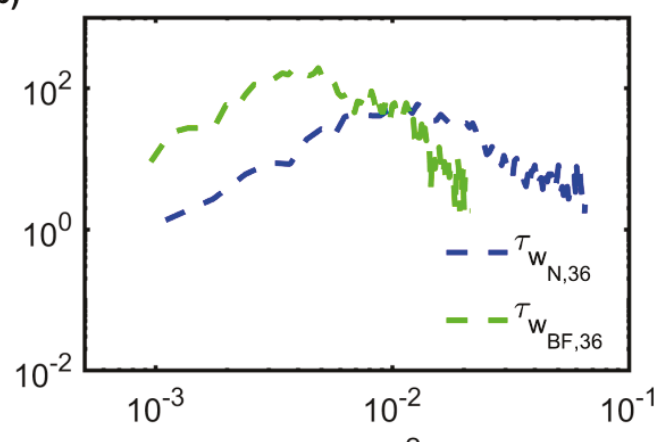

$\tau_{\mathrm{w}}\left(\mathrm{N} / \mathrm{m}^{2}\right)$

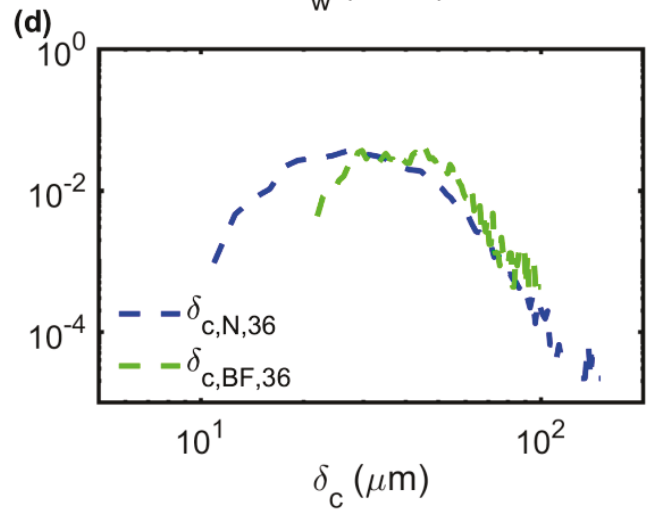

795 Figure 5: PDFs of the pore-scale velocity magnitudes for the clean (a) and bioclogged (b)

796 porous medium. Concentration boundary layer thickness PDFs for the clean (c) and

797 bioclogged (d) porous medium. "BF" and "N" here indicates if the considered data is located

798 within a distance of one pore radius to the biofilm or uncolonized Nafion grains, respectively. 
(a)

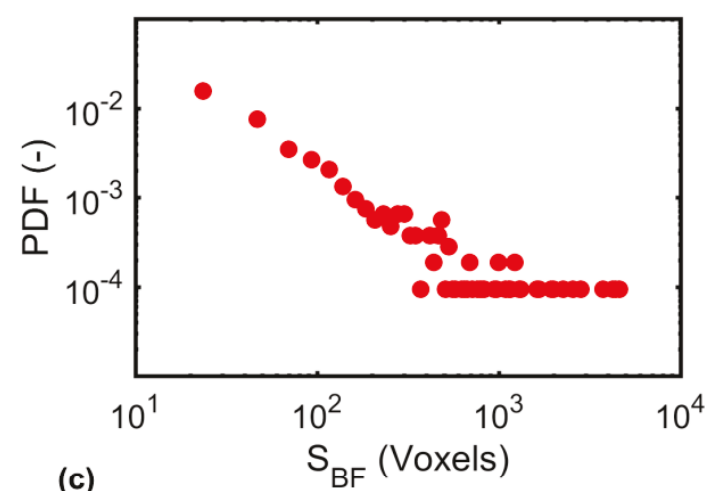

(c)

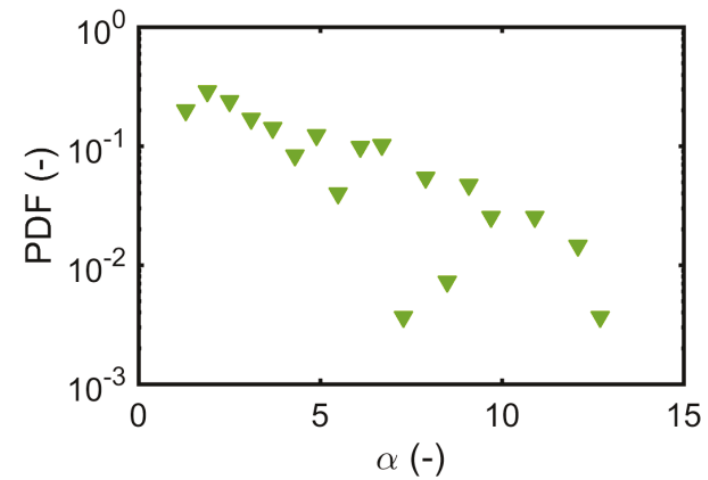

(e)

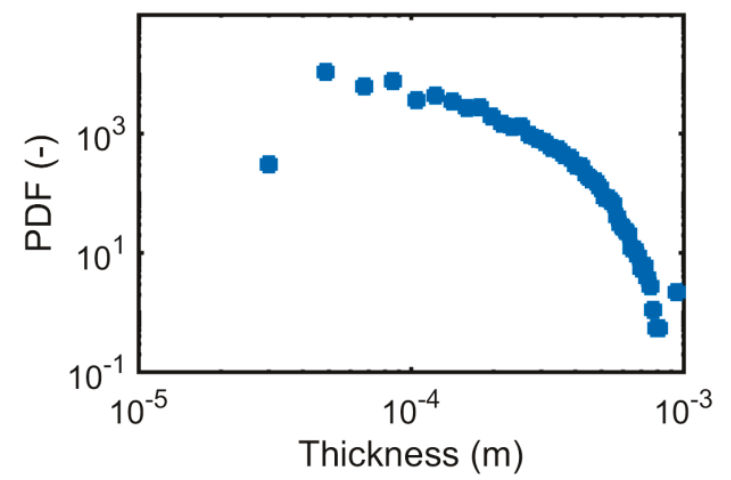

(b)

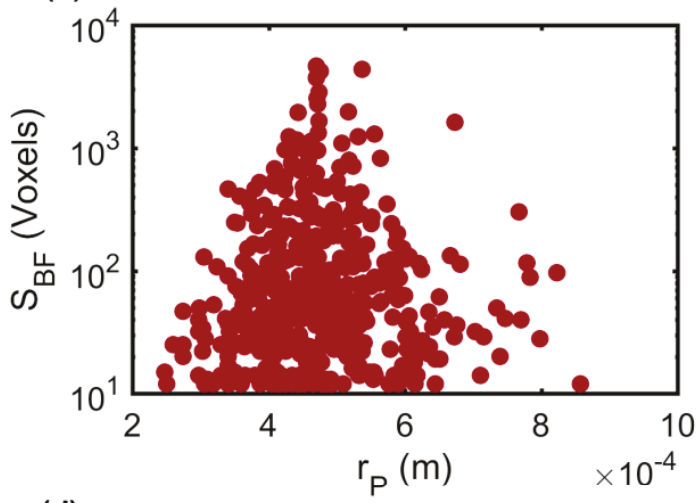

(d)

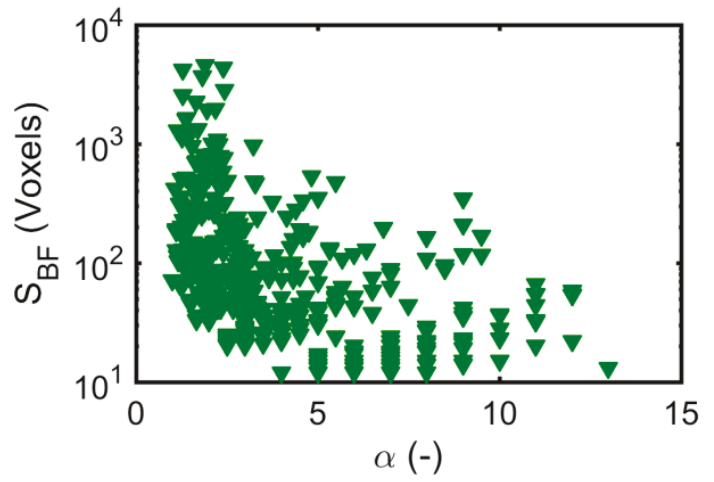

(f)

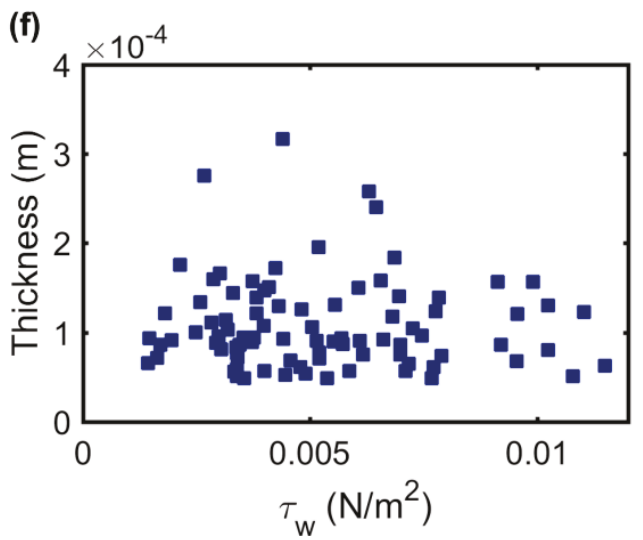

800

Figure 6: (a) PDF of the biofilm patch size $\left(\mathrm{S}_{\mathrm{BF}}\right)$ and (b) correlation of biofilm patch sizes with average pore radii.

(c) PDF of biofilm patch aspect ratio $(\alpha)$ and (d) correlation of 803 biofilm patch size with aspect ratio. (e) PDF of the biofilm thickness and(f) correlation of 804 biofilm thickness with wall shear stress. 
807

808

809

810

811

812

813

814

815

816

817

818

819

820

821

822

823

824

825

826

827 Figure 7: Joint PDFs of $v /\langle v\rangle$ and $r_{P} /\left\langle r_{P}\right\rangle$ for the clean (a) and bioclogged (b) porous media.

828 The dashed and dashed-dotted lines show power laws with exponents of 2 and -2 ,

829 respectively. The white circles are conditional averages of $v /\langle v\rangle$ and $r_{P} /\left\langle r_{P}\right\rangle$ and the

830 continuous line is a power function fitted to the conditional average. The exponents of the

831 power function are indicated as imbedded text.

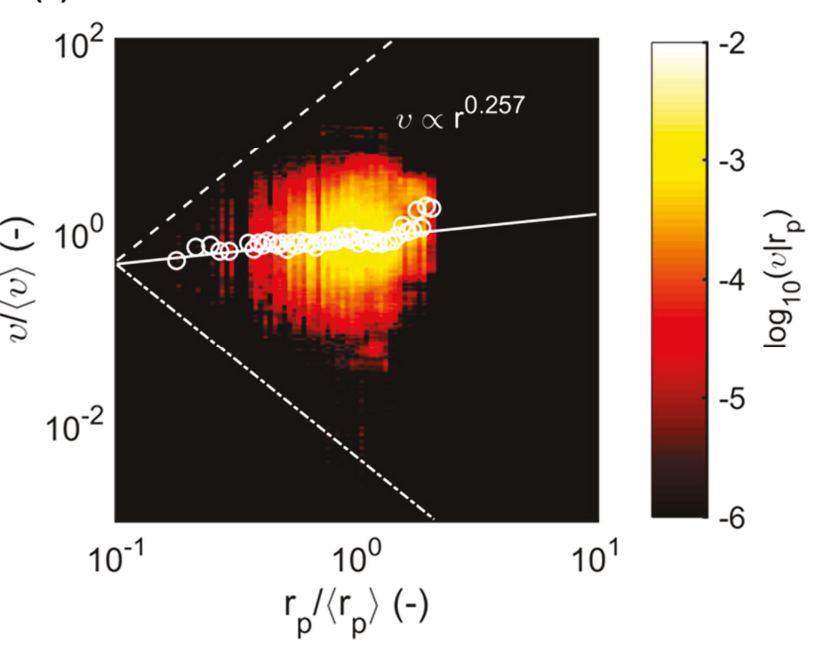

(b)

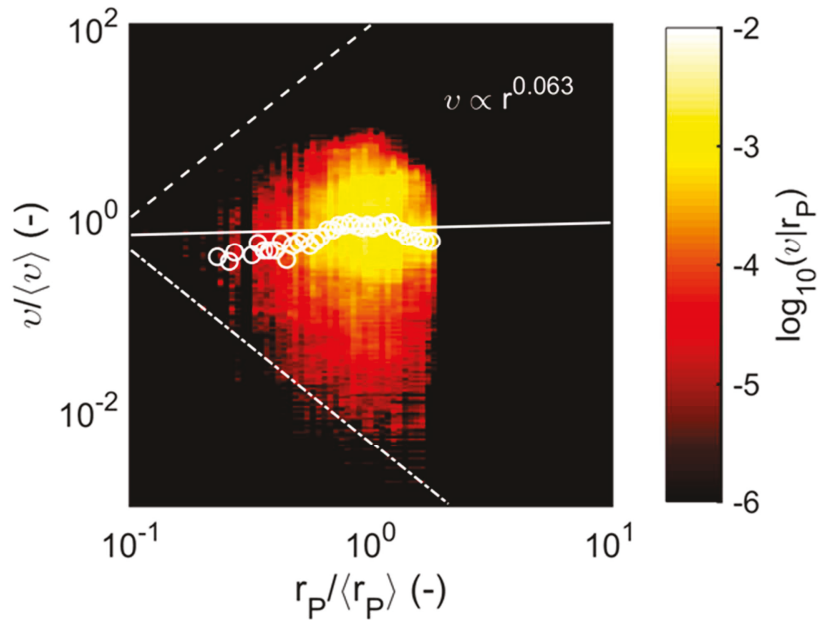




\section{Highlights:}

- Influence of shear and mass transfer on biofilm growth in porous media is studied

- Wide distributions of wall shear stresses and CBL thicknesses measured

- The wall shear stress controls biofilm initial attachment and growth

- Our method allows estimating the biofilm shear for a substratum of complex geometry

- Biofilm growth induces complex changes in the 3D pore-scale hydrodynamics 